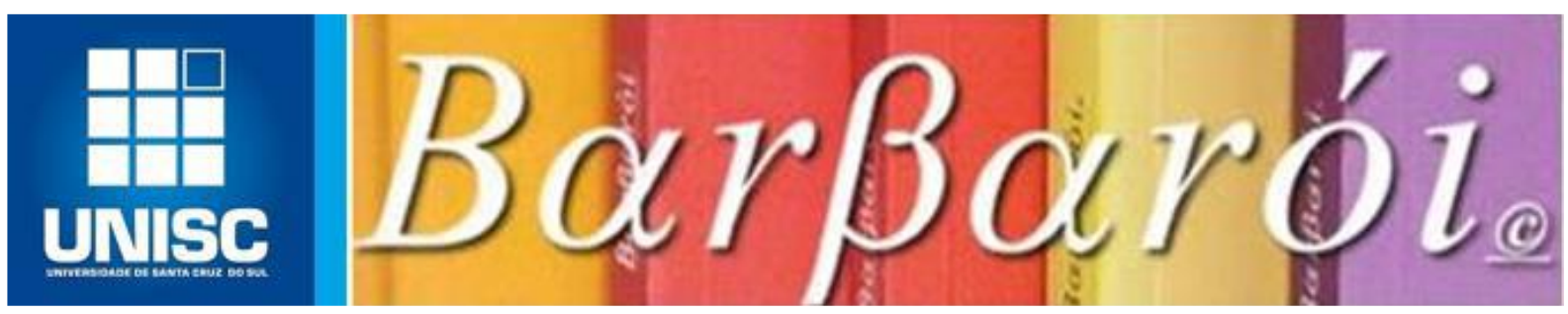

\title{
OBESIDADE, REPRESENTAÇÕES E CATEGORIZAÇÃO SOCIAL
}

DOI: http://dx.doi.org/10.17058/barbaroi.v0i0.14752

\author{
$*$ \\ Ana Maria Justo \\ Universidade Federal de Santa Catarina - UFSC - Brasil \\ Brigido Vizeu Camargo \\ Universidade Federal de Santa Catarina - UFSC - Brasil \\ Andréa Barbará Silva Bousfield \\ Universidade Federal de Santa Catarina - UFSC - Brasil

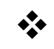

\section{Resumo}

Objetiva-se descrever as representações sociais (RS) e o processo de categorização social referentes à obesidade, a partir de uma abordagem qualitativa. Realizou-se entrevista semidiretiva com 20 homens e 20 mulheres, com e sem excesso de peso e aplicou-se uma escala de silhuetas para avaliar a autoimagem. O processamento dos dados envolveu estatística descritiva e relacional, com o auxílio do SPSS e análise de conteúdo temático-categorial com o auxílio do software ATLAS.Ti, com elaboração de categorias emergentes a partir dos dados e em organização destas em eixos temáticos. Os resultados revelam que a depender da constituição corporal, há diferentes RS e níveis de satisfação com o corpo. A RS relativa à obesidade se organiza em torno da origem, caracterização e consequências, constituindo uma teoria de senso comum sobre o fenômeno em questão. A forte pressão normativa contribui para a emergência de uma RS excessivamente estereotipada. Como consequência, ainda que sejam salientes os riscos de saúde atrelados ao excesso de peso corporal, a tendência é uma não identificação com essa condição, que contribui ao aumento de peso na população.

Palavras-chave: representação social, obesidade, categorização social.

\section{Introdução}

O aumento significativo do peso da população tem sido evidenciado nos globalmente nos últimos anos. Segundo dados da Organização Mundial da Saúde [OMS] (2017), o número de pessoas obesas no mundo mais que dobrou desde a década de 1980. No Brasil, essa proporção ganha destaque e mais metade da população encontra-se com o peso acima do considerado "o ideal”, proporção que aumentou significativamente na década. O percentual de 
obesos subiu de 11,4\% em 2006 para 19,8\% em 2018, e no mesmo período, a proporção de pessoas com sobrepeso avançou de $42,7 \%$ para $55,7 \%$ (BRASIL, 2019).

A OMS (2017) define sobrepeso e obesidade como acúmulo de gordura corporal anormal ou excessiva, que pode ser prejudicial à saúde. Estipula-se que pessoas com Índice de Massa Corporal $\left(\mathrm{IMC}^{1}\right)$ igual ou superior a 25 estão com sobrepeso, e aquelas com IMC igual ou superior a 30 estão obesas. A obesidade é considerada uma doença crônica desde 1975 (OMS, 1997) e mais recentemente configura-se em uma importante questão de Saúde Pública (CASTRO, 2017). A partir disso, uma das estratégias à promoção da saúde da população, bem como prevenção de Doenças Crônicas Não Transmissíveis, lançada pelo Ministério da Saúde, foi a de dedicar-se às políticas que venham cessar o crescimento da proporção de brasileiros acima do peso (BRASIL, 2013).

Ao extrapolar a esfera individual e adentrar o plano sociocultural, os aspectos ligados à obesidade passam a adquirir sentidos que, para além do corpo físico e das características individuais, apontam para suas dimensões sociais (JUSTO \& CAMARGO, 2017). Aqui, procura-se estudar o fenômeno da obesidade não apenas no quadro de uma predisposição a doenças crônicas, tal qual este é encarado pela epidemiologia, mas dentro de um enfoque culturalmente contextualizado, já que o mesmo concentra elementos naturais e simbólicos, privados e sociais e está imerso em um forte contexto normativo. É possível avançar nos conhecimentos sobre o excesso de peso populacional ao procurar compreendê-lo a partir da perspectiva da psicologia social, que, na interface entre a psicologia e a sociologia, pode ser uma ponte para outras áreas do conhecimento (DOISE, 2002).

A partir de representações individuais e sociais, o corpo se configura como uma unidade somatopsíquica, que pode se construir e reconstruir. Conforme Jodelet (2017), o corpo é componente de um pensamento social, regido por sistemas prescritivos, evidenciadas nas cenas sociais. Assim, ele torna-se um objeto privilegiado para se estudar a interação dos processos individuais e coletivos relativos às representações sociais (RS).

Para Moscovici (2012), as RS consistem num tipo de pensamento leigo ou do senso comum, também chamado de pensamento natural (JODELET, 1984) e são formadas para tornar familiar o incomum, permitindo que se dê sentido aos fatos novos ou desconhecidos, tornandoos acessíveis. Ao considerar o conhecimento de senso comum como objeto de estudo,

\footnotetext{
${ }^{1}$ O Índice de Massa Corporal (IMC) é uma relação simples entre o peso e a altura do indivíduo, comumente usada para identificar sobrepeso e obesidade. Calcula-se o IMC dividindo-se o peso da pessoa em quilogramas pelo quadrado de sua altura, em metros (OMS, 2017).
} 
Moscovici considerou o elemento de ligação entre o indivíduo e o mundo que lhe é familiar (ALMEIDA \& SANTOS, 2011). As RS tratam-se de uma forma de interpretar e pensar a realidade cotidiana (JODELET, 1984), de modo que tal conhecimento prático dá um sentido aos eventos cotidianos, fazendo parte da construção social da realidade (BERGER \& LUCKMANN, 1974).

Ao representar determinado objeto, realiza-se uma síntese de diversos fragmentos de saberes e impressões acerca deste, que se organizam entre si, formando uma totalidade coerente. Moscovici (2012) afirma que uma RS é formada articulando três componentes, ou dimensões: (1) informação, que consiste na organização dos conhecimentos que um grupo possui a respeito de um objeto social e pode advir de fontes diversas; (2) atitude, que se trata da orientação global em relação ao objeto da RS; (3) campo ou imagem, que consiste na ideia, imagem, modelo social, no qual há uma unidade hierarquizada de elementos.

As representações sociais criam materialmente os seus objetos, num processo que torna real um esquema conceptual, denominado objetificação. Trata-se da propriedade de tornar concreto o abstrato, de materializar as palavras (JODELET, 1984) e como resultado deste processo, os indivíduos ou grupos atribuem uma realidade física aos conceitos que até então tinham significados abstratos (WAGNER, ELEJABABARRIETA \& LANSTHEINER, 1995).

O processo de objetificação se dá numa relação dialética com o processo de ancoragem, que, segundo Jodelet (1984), comporta o aspecto ligado à integração cognitiva do objeto representado no sistema de pensamentos pré-existentes. As novas representações sobre os objetos do mundo não se inscrevem numa tabula rasa, mas se ancoram em outras representações já existentes. Este processo de enraizamento da representação na vida dos grupos é fundamental aos processos representacionais, pois revela a forma como se dá a sua inserção nos valores de uma determinada cultura ou sociedade.

Tomando o corpo como objeto de representação social, salienta-se que obesidade e magreza sempre estiveram de alguma maneira associados ao belo e ao feio, apesar das transições de significado pelas quais passaram ao longo dos últimos séculos. Até o século XIX, o corpo belo era dotado de formas arredondadas e proeminentes. A abundância foi um marco desse período histórico. Em geral, a gordura era sinônimo de saúde, beleza e sedução. A partir de 1890, todavia, esses valores começaram a se modificar. Estabelece-se, especialmente nas classes médias francesa e norte-americana, a necessidade de ser magro. Nesse período, o estudo da nutrição ganha atenção e ao mesmo tempo os padrões de etiqueta vigoram sobre o consumo alimentar, que deve ser comedido. As revistas de moda passaram a abordar os regimes e 
exercícios físicos, associando a magreza à jovialidade - outro aspecto que já vinha preocupando a população (ANDRADE, 2003; STENZEL, 2002).

O século XIX foi marcado por mudanças, que chegaram ao Brasil um pouco mais tarde, em meados do século XX. Nesse período, as fórmulas milagrosas para perder peso passaram a fazer parte de campanhas publicitárias, e surgia uma nova geração pautada na associação entre saúde, elegância e magreza, que começava a hostilizar o excesso de peso. Também, a medicina no século XX ocupa-se da descrição dos prejuízos da gordura em excesso para a saúde; e a obesidade, que outrora fora atribuída a processos metabólicos, vem a ser associada diretamente à ingestão alimentar em excesso - ou seja: à falta de controle de si e do próprio corpo (JOFFE, 2015; STENZEL, 2002). A conquista da beleza e de um corpo saudável torna-se um objetivo individual, atingido por meio do exercício de autocontrole, envolvendo força de vontade, restrições e vigilância constantes. A gordura, antes associada à saúde, beleza e poder; passou a significar falta de domínio sobre si (ANDRADE, 2003). No século XXI, o corpo magro é o objetivo a ser alcançado em nome da estética, uma escolha social associada ao consumo. A mídia enfatiza o indivíduo saudável, que é equacionado ao "estar em forma" sem "quilos em excesso" (JUSTO \& CAMARGO, 2017).

Conforme Jodelet (2017), o corpo é um mediador do lugar social ocupado pelas pessoas, fornecendo informações que se apresentam em sua aparência e expressividade, por exemplo, as quais se apoiam nas prescrições advindas de grupos e sistemas institucionais. Isso nos leva a pensar no corpo como elemento decisivo nos processos de categorização social. O processo de categorização faz com que se simplifique a realidade, selecionando aspectos do estímulo e agrupando-os numa categoria unificada (TAJFEL, 1981; TORRES, CAMARGO \& BOUSFIELD, 2016). Tal processo está diretamente associado a formação de estereótipos, que são definidos por esses autores como crenças generalizadas, resistentes a mudanças ou novas informações, sobre atributos pessoais de um grupo, simplificando a interpretação do mundo.

É frequente a participação das caraterísticas do corpo nos processos de categorização e generalização, tais como sexo, raça, idade e peso. No que se refere especificamente ao corpo gordo, a literatura indica que pessoas com obesidade ou sobrepeso são comumente atreladas a características tais como: "preguiçoso", "pouco atraente”, "pouco inteligentes”, “compulsivos”, "desmotivados", "gulosos", "auto permissivos", "descontrolados", "inativos", "inseguros", "frustrados" e "indisciplinados" (PUHL, LATNER, KLING, \& LUEDICKE, 2014; LIMA, RAMOS-OLIVEIRA \& BARBOSA, 2017).

O acúmulo de gordura no corpo torna-se um estigma (MATTOS \& LUZ, 2009) e a noção que prevalece é a de que as pessoas gordas ou obesas devem perder peso Barbarói, Santa Cruz do Sul, n. 56, p.<164-188>, jan./jun. 2020 
(BACKSTROM, 2012), uma vez que a gordura corporal representa elementos negativos. As discriminações mostram-se evidentes em distintos domínios da vida em sociedade, tais como empregabilidade, educação e atendimento ao cliente (ARAÚJO, COUTINHO, ARAÚJOMORAIS, SIMEÃO, MACIEL, 2018; BROCHU \& ESSES, 2011). Até mesmo entre os profissionais de saúde, apesar de mencionarem uma preocupação com o problema da obesidade, verifica-se a presença de um conjunto de crenças ambivalentes e de atitudes negativas sobre esses pacientes (LIMA, RAMOS-OLIVEIRA \& BARMOSA, 2017).

Sudo e Luz (2007) distinguem que na maioria das vezes o "ser gordo" não é pautado pelo peso na balança, mas pela noção subjetiva do estar acima do peso. O corpo objetivo é percebido por meio do corpo subjetivo e essa experiência vem modificar a imagem corporal, a estima de si mesmo e a relação com o corpo do outro (ANDRIEU, 2006). A imagem corporal, para Schilder (1999), é a representação mental que um indivíduo tem do seu corpo. É multifacetada, incorporada pela percepção, mas também por dimensões afetivas, cognitivas e comportamentais (BANFIELD \& MCCABE 2002; LEGENBAUER, RÜHL \& VOCKS, 2008). Neste sentido, Lopes (2007) propõe que ao falar da imagem, não se fala apenas de um corpo anatômico, real e objetivo, instrumento de funções de locomoção, de apreensão e sinestesia, mas de uma corporeidade, a qual faz referência à realidade subjetiva. Fala-se de um corpo que, tendo atravessado experiências diferentes durante a vida, conta uma história, inscrita na própria imagem.

A percepção subjetiva que uma pessoa tem sobre seu corpo pode ser mais importante do que a realidade objetiva de sua aparência. Nesse sentido, o peso, por si só, não parece ser um único determinante do grau de satisfação com a imagem corporal (LIRA, ET AL, 2017). No estudo de Camargo, Justo e Jodelet (2010), a insatisfação corporal mostrou-se mais associada à dependência em relação ao olhar e julgamento do outro, do que com o corpo real dos participantes, reiterando o predomínio dos elementos sociais subjetivos na formação da autoimagem.

Observa-se, a partir da revisão de literatura, que a obesidade é marcada por uma visão negativa e marginalizada (ARAÚJO et al., 2018; SUDO \& LUZ, 2007; WOTT \& CARELS, 2010). Os padrões relativos a um corpo belo e saudável, vão na contramão do acúmulo de quilos em excesso e os estudos recentes apontam uma representação estereotipada acerca do que é ser obeso. Ao mesmo tempo, sabe-se que o número de pessoas obesas e com sobrepeso tem aumentado consideravelmente, sendo que no Brasil o grupo de pessoas acima do peso considerável saudável correspondente a mais de $50 \%$ da população (BRASIL, 2019). Assim, questiona-se a respeito de quais são as crenças relativas à obesidade que circulam em meio à Barbarói, Santa Cruz do Sul, n. 56, p.<164-188>,jan./jun. 2020 
população. Para tanto, pretende-se identificar o processo de categorização social relativo à obesidade, assim como descrever as representações sociais ligadas a ela, por meio de um estudo qualitativo com homens e mulheres de diferentes composições corporais.

\section{Método}

Para atingir o objetivo proposto, realizou-se uma pesquisa qualitativa, do tipo levantamento de dados, de natureza descritiva e comparativa.

\section{Participantes}

Participaram deste estudo 20 homens e 20 mulheres, trabalhadores da região sul do Brasil com idade entre 30 e 57 anos [M = 38 anos e 5 meses; DP $=7$ anos e 9 meses]. Todos os participantes tiveram o IMC calculado a partir do peso e altura auto referidos ao pesquisador durante a entrevista e então foram divididos em dois grupos pareados: com excesso de peso e sem excesso de peso $^{2}$. O primeiro grupo de participantes teve média de IMC igual a 31,44 [DP $=6,60]$ enquanto o grupo de participantes sem excesso de peso teve a média de IMC igual a $22,67[\mathrm{DP}=2,62]$. Os participantes foram recrutados em instituições de trabalho vinculadas à prefeitura de uma capital da região sul do Brasil. Como critérios de inclusão foram considerados: concordância em participar, não exercer atividade profissional relacionada ao tema do estudo e estar na faixa etária entre 30 e 59 anos. Sobre o último critério, salienta-se que é nesta faixa de idade que as pessoas estão mais propensas ao ganho de peso (PEIXOTO et al., 2017). Após a realização da entrevista solicitava-se que os participantes indicassem outros possíveis colaboradores com a pesquisa, criando-se dessa maneira uma rede de contatos que viabilizava o acesso aos futuros entrevistados.

\section{Técnica de coleta de dados}

Foi utilizada a técnica de entrevista semi-diretiva (GHIGLIONE \& MATALON,1993), a qual permite ao pesquisador acessar representações subjetivas do entrevistado, para além dos conteúdos fatuais. A entrevista era desenvolvida a partir de perguntas norteadoras, que buscaram contribuir com os objetivos de pesquisa, por meio da técnica de entrevista semidiretiva (GASKELL, 2002): O que você sabe sobre a obesidade? O que você acha das pessoas

\footnotetext{
${ }^{2}$ Como critério de corte, entre as mulheres considerou-se sem sobrepeso aquelas com IMC $<25,0$, mesmo critério utilizado pela OMS (2017) e para os homens este critério foi adaptado, sendo considerados com excesso de peso aqueles com o IMC superior a 26,5, valor que mostra-se mais condizente com a constituição corporal dos brasileiros, onde um maior massa não necessariamente este associada ao acúmulo de gordura, mas ao desenvolvimento muscular, amplamente valorizado pela cultura brasileira entre os homens.
}

Barbarói, Santa Cruz do Sul, n. 56, p.<164-188>, jan./jun. 2020 
que estão acima do peso? E o que você acha que a sociedade pensa a respeito disso? Em sua opinião, o que define que uma pessoa é gorda?

Ao final da entrevista, os participantes respondiam a uma escala de silhuetas (KAKESHITA, SILVA, ZANATTA, \& ALMEIDA, 2009) com validade e fidedignidade adequadas à população brasileira, para avaliação da autoimagem e imagem ideal e informavam seu peso e altura (para cálculo do IMC), assim como informavam algumas questões para a caracterização da amostra. Todas as entrevistas de pesquisa foram gravadas em sua íntegra e posteriormente transcritas. Ao final da participação houve um momento de dessensibilização, no qual o participante era convidado a falar livremente sobre o assunto ou esclarecer dúvidas, visando minimizar possíveis efeitos negativos da entrevista. O projeto obteve parecer favorável do Comitê de Ética em Pesquisa com Seres Humanos (CAAE: 20712613.6.0000.0121; Parecer n. 447.940) e todas as diretrizes éticas foram seguidas. Todos os participantes eram voluntários e assinavam ao Termo de Consentimento Livre e Esclarecido

\section{Análise dos dados}

Os dados referentes à caracterização da amostra, IMC e escalas de silhuetas foram submetidos a análises estatísticas descritivas e relacionais, com o auxílio do software SPSS (versão 17.0). Já o conteúdo textual das entrevistas foi submetido à análise de conteúdo temático-categorial.

A análise de conteúdo temático-categorial foi realizada com o auxílio do software ATLAS TI (MUHR, 2004) para a sistematização dos dados e se deu a partir da execução das fases de pré-análise, tratamento dos dados e síntese dos resultados (BARDIN, 2009), com elaboração de categorias emergentes a partir dos dados e em seguida organizadas em eixos temáticos.

\section{Resultados}

\section{IMC e Imagem corporal}

Conforme o delineamento do estudo, os 40 entrevistados foram divididos em grupos, conforme sexo e classificação de IMC. Além das medidas antropométricas auto-atribuídas, foi apresentada aos participantes uma escala de silhuetas (KAKESHITA et al, 2009) e solicitavase que os mesmos escolhessem duas imagens: uma que representasse seu corpo da maneira como ele se via naquele momento (autoimagem corporal) e outra que representasse o corpo que ele ou ela gostariam de ter (imagem ideal). As silhuetas foram classificadas de acordo com a tabela de IMC da OMS (2017), em baixo peso, peso normal, sobrepeso e obesidade. Ao 
cruzarem-se as informações de peso e altura declarados com os resultados da escala de silhuetas, conforme a maior parte das pessoas sem excesso de peso identificou-se assim na escala de silhuetas, bem como quase todos os com excesso de peso assim se identificaram na escala.

A partir das informações explicitadas na Tabela 1, com o comparativo das medidas de IMC e de autoimagem entre as pessoas com e sem excesso de peso (EP) e homens e mulheres, verifica-se que houve diferença significativa de IMC entre os grupos com e sem excesso de peso $[\mathrm{t}=5,52 ; \mathrm{gl}=38 ; \mathrm{p}<0,001]$ garantindo o delineamento previsto no estudo. Constatou-se que o IMC foi mais influente nas medidas de autoimagem do que o sexo dos participantes. A silhueta percebida tem a média quase cinco pontos mais alta entre os participantes com excesso de peso [t=6,76; $\mathrm{gl}=38 ; \mathrm{p}<0,001]$, a silhueta considerada ideal é quase dois pontos maior entre estes participantes $[\mathrm{t}=3,82 ; \mathrm{gl}=38 ; \mathrm{p}<0,001]$ e a insatisfação corporal chega a ser 3 pontos maior $[\mathrm{U}=24,5 ; \mathrm{Z}=4,84 ; \mathrm{p}<0,001]$.

Tabela 1

Descrição das variáveis e escalas relativas ao IMC e autoimagem corporal

\begin{tabular}{ccccccc}
\hline \multirow{2}{*}{ Variáveis ou escalas } & & \multicolumn{2}{c}{ Peso } & \multicolumn{2}{c}{ Sexo } \\
\cline { 3 - 7 } & & Geral & Sem EP & Com EP & Homens & Mulheres \\
\hline \multirow{2}{*}{ IMC } & $\mathrm{M}$ & 27,06 & $22,67^{* *}$ & $31,44^{* *}$ & 28,54 & 25,56 \\
& $\mathrm{~N}$ & 6,65 & 2,62 & 6,60 & 7,68 & 5,21 \\
\multirow{2}{*}{ Silhueta percebida } & $\mathrm{M}$ & 7,33 & $4,95^{* *}$ & $9,70^{* *}$ & 7,55 & 7,10 \\
& $D P$ & 3,25 & 2,11 & 2,32 & 3,22 & 3,35 \\
Silhueta ideal & $\mathrm{M}$ & 5,72 & $4,70^{* *}$ & $6,55^{* *}$ & 5,95 & 5,30 \\
& $\mathrm{~N}$ & 1,78 & 1,72 & 1,32 & 1,93 & 1,59 \\
Insatisfação corporal * & $\mathrm{M}$ & $-1,70$ & $-0,25^{* *}$ & $-3,15^{* *}$ & $-1,60$ & $-1,80$ \\
& $D P$ & 2,03 & 1,12 & 1,66 & 1,87 & 2,21 \\
\hline
\end{tabular}

*Esta variável não apresentou distribuição normal $(\mathrm{K}-\mathrm{S}=0,16$; gl=40; $\mathrm{p}<0,05)$ e para ela utiliza-se o teste não paramétrico de comparação de médias do tipo Mann Whitney. Para as demais variáveis, utilizou-se o teste T. $* * \mathrm{p}<0,001$.

Verifica-se que a diferente constituição corporal reflete em distintas representações e satisfação relativas ao próprio corpo, e também no que é considerado um corpo ideal. Se por um lado as pessoas com excesso de peso apresentam maior nível de insatisfação com a silhueta, as mesmas também se mostram menos exigentes em termos da silhueta que consideram o corpo ideal. Por outro lado, ao contrário do que aponta a literatura, não foram constatadas diferenças significativas entre homens e mulheres.

\section{Categorização social: o que define que uma pessoa é gorda?}

Buscou-se identificar junto aos participantes quais os critérios seriam usados para caracterizar uma pessoa como "gorda". As respostas passaram por uma análise de conteúdo temático-categorial e a síntese dos resultados encontrados apresenta-se na Tabela 2, podendoBarbarói, Santa Cruz do Sul, n. 56, p.<164-188>,jan./jun. 2020 
se facilmente constatar que os critérios objetivos prevalecem significativamente, embora tenham sido apontados também critérios subjetivos.

Tabela 2.

Análise de conteúdo - Em sua opinião, que define que uma pessoa é gorda?

\begin{tabular}{|c|c|c|c|c|c|}
\hline Grandes temas & Categorias & Elementos temáticos & $\begin{array}{c}\text { Sem } \\
E P\end{array}$ & $\begin{array}{c}\text { Com } \\
E P\end{array}$ & Geral. \\
\hline \multirow{14}{*}{$\begin{array}{l}\text { Critérios objetivos } \\
\text { definem que uma } \\
\text { pessoa é gorda }\end{array}$} & \multirow{7}{*}{ Aparência $(n=131)$} & visual & 26 & 15 & 41 \\
\hline & & partes do corpo & 23 & 13 & 35 \\
\hline & & gordura & 16 & 5 & 21 \\
\hline & & roupas & 8 & 4 & 12 \\
\hline & & estética & 4 & 5 & 9 \\
\hline & & idade & 5 & 2 & 7 \\
\hline & & chama atenção & 5 & 1 & 6 \\
\hline & \multirow{4}{*}{ Medida $(\mathrm{n}=83)$} & relação peso/altura & 17 & 19 & 36 \\
\hline & & tamanho & 12 & 9 & 21 \\
\hline & & medida & 10 & 10 & 20 \\
\hline & & médico & 3 & 3 & 6 \\
\hline & \multirow[t]{2}{*}{ Limitações $(n=25)$} & limitação física & 7 & 9 & 16 \\
\hline & & limitação social & 3 & 0 & 3 \\
\hline & Obesidade extrema $(n=5)$ & obesidade extrema & 2 & 3 & 5 \\
\hline \multirow{5}{*}{$\begin{array}{l}\text { Critérios subjetivos } \\
\text { definem que uma } \\
\text { pessoa é gorda }\end{array}$} & \multirow{3}{*}{ Padrões sociais $(n=47)$} & padrões & 13 & 26 & 39 \\
\hline & & mulheres cobradas & 5 & 0 & 5 \\
\hline & & olhar do outro & 0 & 3 & 3 \\
\hline & É relativo $(n=14)$ & relativo & 11 & 3 & 14 \\
\hline & Fatores psi $(n=4)$ & fatores psicológicos & 1 & 3 & 4 \\
\hline
\end{tabular}

Os critérios subjetivos de julgamento estão associados principalmente aos padrões que são impostos socialmente: "O que define, não só para mim, mas para muitas outras pessoas são os padrões que são estabelecidos. São as convenções, os padrões que a sociedade estabeleceu como certo e como errado" (P7, Homem, com excesso de peso). Dentro dessa concepção, o "ser gordo" depende do contexto que o indivíduo está inserido. "Depende do conceito geral de gordo que a sociedade adota" (P38, Homem, sem excesso de peso). Ademais, houve a menção a fatores psicológicos, como por exemplo, a autoimagem, para caracterizar o fenômeno: “Geralmente a pessoa está obesa psicologicamente, mas fisicamente ela está bem. Onde eu quero chegar, eu quero chegar ao seguinte, a obesidade não está só no sentido físico da coisa" (P11, Homem, sem excesso de peso). Este participante refere-se àquelas pessoas que embora tenham um peso dentro dos parâmetros considerados saudáveis, não estão satisfeitas com seu corpo, sentindo ser "gordas". 
Já os critérios objetivos - que foram mencionados em larga maioria; não discutem qualquer norma ou padrão estético como convenção social. Ao contrário, a norma está intrínseca ao discurso, que cita principalmente a aparência do corpo como o critério de identificação da gordura. “Como eu sei, é enxergando. É vendo. Não tem como você saber só pela voz se uma pessoa é gorda ou magra, você tem que ver. É pelo físico. Creio que é só pelo físico, porque a gordura aparece no físico" (P13, Mulher, sem excesso de peso). Partes do corpo específicas (principalmente a barriga, os braços e as pernas) são indicados, assim como a gordura aparente: "Quando ela tem mais massa gorda no corpo do que massa magra, mais gordura no corpo do que pode, eu acho que é isso que define" (P12, Mulher, sem excesso de peso).

Fala-se também em extrapolar um determinado limite de peso, como no excerto a seguir: "Mas eu acho que definir o que é uma pessoa gorda, para mim é isso, estar nesse excesso[...] que se passe de um limite da normalidade, daquilo que seria o normal para cada um de nós" (P26, Mulher, com excesso de peso). Este limite é estabelecido, segundo alguns participantes, por meio da relação entre peso e altura "O que define? Eu acho que é aquela, aquelas famosas tabelinhas, que tem a massa corporal relacionado à altura da pessoa" (P32, Mulher, com excesso de peso).

As limitações físicas e/ou sociais também aparecem como critério objetivo, ainda que em menor frequência que as características estéticas. As limitações físicas envolvem a perda da funcionalidade do corpo e são evocadas dificuldades em se locomover, realizar atividades cotidianas, cansaço excessivo, dificuldade em respirar. "É alguém que não consegue amarrar o cadarço de tênis, não consegue colocar uma meia e aí vai" (P5, Mulher, excesso de peso). Por sua vez as limitações sociais envolvem a ausência das pessoas gordas em determinados espaços, ou a dificuldade encontrada por elas para viver tais situações de interação: "É difícil tu ver um gordo na praia, porque a tendência é se esconder. O gordo que vai para a praia, assumido, eu acho bacana até, bem resolvido. Mas eu tenho dúvidas de que um gordo seja bem resolvido" (P13, Mulher, sem excesso de peso). Por fim, aparece o gordo ligado a casos extremos, no que segundo os critérios médicos seria definido como obesidade extrema: "Aquele super obeso que já não tem mais jeito, só com a cirurgia" (P36, Homem, excesso de peso), associado à necessidade de intervenções médicas invasivas.

\section{Obesidade: uma teoria de senso comum}

Com o objetivo de explorar mais especificamente os conteúdos representacionais relativos à obesidade, visto que este se trata do principal objetivo do estudo, foi realizada uma Barbarói, Santa Cruz do Sul, n. 56, p.<164-188>,jan./jun. 2020 
análise de conteúdo temático-categorial dos fragmentos das entrevistas que abordavam diretamente o tema da obesidade. A Tabela 3 apresenta as categorias encontradas na análise de conteúdo temática.

Tabela 3

Análise de conteúdo - RS da Obesidade

\begin{tabular}{|c|c|c|c|c|c|}
\hline Grandes Temas & Temas & Códigos & Sem EP & Com EP & Geral \\
\hline \multirow{15}{*}{ 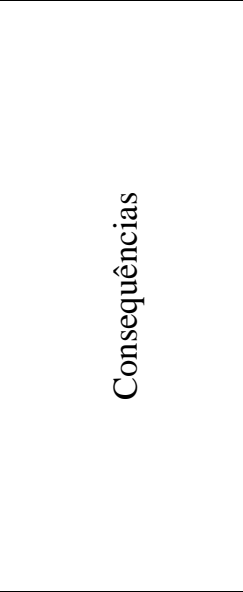 } & \multirow{7}{*}{ Físicos } & Problemas de saúde & 14 & 30 & 44 \\
\hline & & Limitações físicas & 8 & 13 & 21 \\
\hline & & Morte & 2 & 2 & 4 \\
\hline & & Incapacidade & 1 & 2 & 3 \\
\hline & & Dificuldades sono & 1 & 2 & 3 \\
\hline & & Futuro & 0 & 2 & 2 \\
\hline & & & 26 & 51 & 77 \\
\hline & \multirow{4}{*}{ Sociais } & Preconceito & 7 & 21 & 28 \\
\hline & & Restrição social & 5 & 12 & 17 \\
\hline & & Empregabilidade & 2 & 2 & 4 \\
\hline & & & 14 & 35 & 49 \\
\hline & \multirow{3}{*}{ Psicológicos } & Autoestima & 7 & 6 & 16 \\
\hline & & Dificuldades psicológicas/emocionais & 5 & 10 & 15 \\
\hline & & & 12 & 16 & 31 \\
\hline & \multicolumn{2}{|c|}{ Total Consequências } & 52 & 102 & 154 \\
\hline \multirow{15}{*}{ 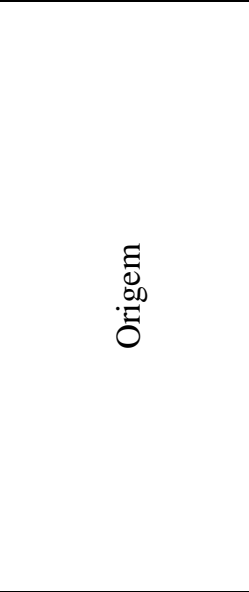 } & \multirow{5}{*}{ Comportamental } & Não se cuidam & 19 & 10 & 29 \\
\hline & & Alimentação & 13 & 9 & 22 \\
\hline & & Sedentarismo & 7 & 3 & 10 \\
\hline & & & 39 & 22 & 61 \\
\hline & & Psicológico/Emocional & 8 & 17 & 25 \\
\hline & \multirow{5}{*}{ Fisiológico } & Genética & 6 & 7 & 13 \\
\hline & & Hormonal & 3 & 5 & 8 \\
\hline & & Medicamento & 1 & 2 & 3 \\
\hline & & Idade & 1 & 1 & 2 \\
\hline & & & 11 & 15 & 26 \\
\hline & \multirow{4}{*}{ Circunstancial } & Família & 5 & 4 & 9 \\
\hline & & Falta Estímulo & 1 & 3 & 4 \\
\hline & & Falta tempo & 0 & 2 & 2 \\
\hline & & & 6 & 9 & 15 \\
\hline & \multicolumn{2}{|l|}{ Total Origem } & 64 & 63 & 127 \\
\hline \multirow{18}{*}{ 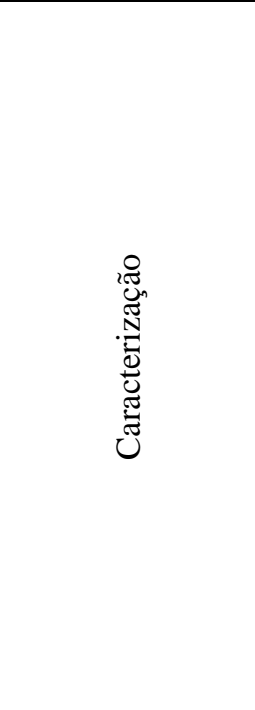 } & \multirow{7}{*}{ Imagem } & Criança & 11 & 4 & 15 \\
\hline & & Obesidade extrema & 6 & 6 & 12 \\
\hline & & Acentos especiais & 3 & 4 & 7 \\
\hline & & IMC & 2 & 3 & 5 \\
\hline & & Gordura & 1 & 1 & 2 \\
\hline & & Força & 0 & 1 & 1 \\
\hline & & & 23 & 19 & 42 \\
\hline & \multirow{6}{*}{ Descrição } & Doença & 7 & 13 & 20 \\
\hline & & Problema sério & 6 & 5 & 11 \\
\hline & & Comum & 1 & 1 & 2 \\
\hline & & Igual sobrepeso & 5 & 2 & 7 \\
\hline & & Diferente gordo & 1 & 0 & 1 \\
\hline & & & 20 & 21 & 41 \\
\hline & \multirow{4}{*}{ Negativo } & Ninguém quer ser & 1 & 5 & 6 \\
\hline & & Negativo & 1 & 5 & 6 \\
\hline & & Repugna & 2 & 0 & 2 \\
\hline & & & 4 & 10 & 14 \\
\hline & \multicolumn{2}{|c|}{ Total Caracterização } & 47 & 50 & 97 \\
\hline \multirow{2}{*}{ 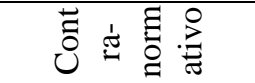 } & \multirow{2}{*}{ Emagrecimento } & Redução estômago & 8 & 8 & 16 \\
\hline & & Tratamento médico & 2 & 7 & 9 \\
\hline
\end{tabular}

Barbarói, Santa Cruz do Sul, n. 56, p.<164-188>,jan./jun. 2020 


\begin{tabular}{|c|c|c|c|c|c|}
\hline & & $\begin{array}{l}\text { Não consegue perder } \\
\text { Precisa de ajuda } \\
\text { Tratamento psicológico }\end{array}$ & $\begin{array}{l}7 \\
2 \\
1 \\
\mathbf{2 0} \\
\end{array}$ & $\begin{array}{l}7 \\
5 \\
3 \\
\mathbf{3 0} \\
\end{array}$ & $\begin{array}{l}14 \\
7 \\
4 \\
\mathbf{5 0}\end{array}$ \\
\hline & & Mídia & 4 & 8 & 12 \\
\hline & & Roupa & 4 & 5 & 9 \\
\hline & Norma social & $\begin{array}{l}\text { Padrão de beleza } \\
\text { Estética } \\
\text { Cultura }\end{array}$ & $\begin{array}{l}2 \\
0 \\
1 \\
\mathbf{1 1}\end{array}$ & $\begin{array}{l}5 \\
6 \\
0 \\
\mathbf{2 4}\end{array}$ & $\begin{array}{l}7 \\
6 \\
1 \\
35\end{array}$ \\
\hline & Total Contra-norn & ativo & 31 & 54 & 85 \\
\hline \multirow[t]{2}{*}{$\stackrel{\substack{0 \\
ٍ}}{0}$} & \multirow[t]{2}{*}{ Minha experiência } & $\begin{array}{l}\text { Não me sinto obeso } \\
\text { Não conheço } \\
\text { Difícil Falar } \\
\text { Cheguei na obesidade }\end{array}$ & $\begin{array}{l}0 \\
0 \\
0 \\
0 \\
\mathbf{0}\end{array}$ & $\begin{array}{l}2 \\
1 \\
1 \\
4 \\
\mathbf{8}\end{array}$ & $\begin{array}{l}2 \\
1 \\
1 \\
4 \\
8\end{array}$ \\
\hline & & Aceitação social & 7 & 3 & 10 \\
\hline
\end{tabular}

A análise de conteúdo permite afirmar que o que predomina na fala dos participantes em relação à obesidade são as consequências negativas da mesma, principalmete no que se refere aos agravos à saúde e mobilidade. "É como eu te falei, a minha primeira impressão é que eu fico preocupada, porque me parece que elas [as pessoas obesas] não têm saúde" (P32, mulher com excesso de peso). No entanto, também são importantes as consequências associadas às perdas nas relações sociais, o que envolve o preconceito sofrido e as restrições, inclusive no que se refere à busca por um emprego. Por fim, são mencionadas as consequências psicológicas e/ou emocionais da obesidade, marcadamente negativas. Destaca-se que houve maior menção às consequências da obesidade entre os particpantes com sobrepeso, os quais evocaram o dobro de elementos em relação aos participantes sem sobrepeso. Embora a maiora destes não seja clinicamente obeso, considera-se que possa haver maior implicação com o tema em questão. “Acredito eu que para quem está acima do peso, para quem é obeso, é complicado. Acho que é complicado conviver com isso em todos os sentidos... pelo preconceito enfrentado, mas por questões de saúde também" (P7, homem com excesso de peso).

A segunda dimensão mais abordada pelos participantes foi a origem da obesidade, o que esteve presente em 127 das unidades analisadas. As causas dividiram-se em comportamentais, psicológicas, fisiológicas e circunstanciais. É evidente o destaque da origem comportamental, que envolve má alimentação, sedentrarismo e até mesmo um "descuido" do próprio corpo, origem esta que é mais frequentemente mencionada pelos participantes sem excesso de peso. "Então ela vai comer o que tiver de mais prático, coxinha, refrigerante, essas coisas, isso que traz muito mais peso" (P9, mulher sem excesso de peso); trecho que evidencia a culpabilização individual, responsabilizando diretamente a pessoa obesa por seus comportamentos alimentares inadequados, os quais seriam realizados por maior praticidade ou acessibilidade. 
A origem psicológica/emocional, por sua vez é mais evidente entre os participantes com excesso de peso e pode ser compreendida a partir do excerto a seguir:

“...um problema psicológico, uma depressão pode trazer a obesidade a uma pessoa.
Ela é mais uma consequência do que uma causa, muitas vezes. Então ela deveria ser
tratada como uma consequência, e serem investigadas as causas. O que se faz
geralmente é o contrário, se ataca a obesidade achando que ela é uma causa. Na
verdade, ela é muitas vezes uma consequência de alguma coisa que a pessoa está
passando. Hoje é muito mais fácil ficar isolado no meio de uma multidão do que
sozinho em casa. Acaba contribuindo para muitos quadros de obesos” (P21, homem
com excesso de peso).

A ênfase na origem fisiológica da obesidade aparece também, com frequência semelhante à origem psicológica e evidencia, sobretudo a genética e as disfunções hormonais. Por fim, verifica-se que a atribuição de causa externa, ou circunstancial foi a menos mencionada e apresentou uma origem no contexto familiar e seus hábitos, falta de estímulo esterno para o autocuidado ou falta de tempo decorrente da rotina diária.

Uma terceira dimensão presente na RS da obesidade é a caracterização da mesma, em termos de imagem, descrição e avaliação. A imagem salienta, principalmente para aqueles sem excesso de peso, a obesidade infantil, onde imediatamente as pessoas falam das crianças ao pensar em obesidade: “Agora está aqui, aqui está todo mundo gordinho, as crianças, tudo obesas" (P30, mulher, sem excesso de peso). Essa imagem se aproxima da estereotipia, onde a obesidade é associada às pessoas morbidamente obesas, que necessitam de espaços especializados. "Porque assim, eu estou falando em obeso daquele obeso bem, bem, bem, bem..." (P32, mulher com excesso de peso). "Vem a figura da pessoa extremamente gorda e triste que se isola, tu vês aqueles obesos que estão em casa, pesam quase 300 quilos e nem se mexem direito, dependem dos outros. Já tenho essa visão quando fala em obesidade. Vem um gordo gigante na mente, extremamente gordo" (P36, homem sobrepeso).

Em geral, verifica-se que a obesidade é associada a uma doença ou um problema muito sério. "A obesidade é uma coisa para mim maior, é um problema de saúde mesmo" (P29, mulher com excesso de peso), e carregada de afetos negativos; "Eu penso que a obesidade se resumiria a isso, uma coisa muito negativa para a pessoa, muito mais do que o sobrepeso" (P39, homem, com excesso de peso), sendo raramente identificada como algo que passa a fazer parte da nossa sociedade (comum $n=2$ ).

Tal caracterização da obesidade reflete no próximo tema abordado que é a sua dimensão contra normativa, ou seja, a necessidade de reverter a situação de obesidade, logo, a necessidade de emagrecer; e o quanto ela está na contramão dos padrões estéticos presentes na cultura atual e difundidos pela mídia. Dentro dessa dimensão, os obesos são pessoas que não conseguem diminuir o peso e que teriam a necessidade de enfrentar diferentes tipos de intervenções médicas Barbarói, Santa Cruz do Sul, n. 56, p.<164-188>,jan./jun. 2020 
e/ou psicológicas para ter sucesso no seu emagrecimento, sendo que dentre elas a redução de estômago aparece como uma solução possível. Essas ideias aparecem com maior frequência dentre os participantes com excesso de peso.

Mostra-se nas falas dos participantes a norma social predominante, que envolve a mídia e a moda, as quais veiculam os padrões de beleza presentes em nossa cultura que se mostram justamente como uma oposição à obesidade.

\footnotetext{
“A sociedade pensa o que os padrões de beleza impõem...Ela é influenciada por padrões de consumo, por padrões de beleza que são impostos a ela, desde um outdoor, você vê um outdoor de um condomínio para vender, você não vê uma família gorda. Vê pessoas magras, bonitas, loiras de olhos azuis. Ou você não vê uma pessoa gorda. Eu não sei, se eu fosse obeso ou gordo eu estaria muito mal. É uma situação muito chata" (P3, homem com excesso de peso).
}

Por fim, observam-se os participantes com excesso de peso falando de sua experiência, sentindo-se ou não obesos, falando sobre pessoas obesas e o considerando um assunto difícil a ser abordado por um participante obeso. Em contrapartida, houve 10 menções à aceitação da condição da obesidade, como uma condição que deve ser aceita sem críticas ou necessidade de mudança; sendo sua maior parte entre participantes sem excesso de peso.

\section{Discussão}

A partir do cálculo do IMC dos participantes foram identificados dois grupos distintos, de acordo com a relação atribuída entre seu peso e altura, segundo os critérios propostos pela OMS (2017). É preciso salientar que quando aqui fala-se em grupo, este não se caracteriza como um "verdadeiro grupo social" (CAMPOS, 2014). O grupo nessa pesquisa faz alusão ao conceito de categoria social, tal qual descrito por Jesuíno (2002), em que são considerados os indivíduos com algum atributo em comum que assim diferencia-os de outras categorias (CAMPOS, 2014). No caso, utilizou-se a constituição corporal, por meio de IMC calculado a partir de peso e altura auto atribuídos pelos participantes no momento da coleta dos dados, formando grupos taxonômicos.

No que diz respeito à validade do cálculo de IMC a partir de peso e altura auto referidos, Peixoto, Benício e Jardim (2006) apontam que embora seja constatada uma pequena subestimação do IMC dos participantes a partir de medidas antropométricas auto atribuídas, a mesma não possui significância capaz de inviabilizar seu uso, a qual foi considerada válida para avaliar a presença de excesso de peso corporal na população brasileira.

Além disso, a medida da silhueta percebida por meio de uma escala de silhuetas (KAKESHITA et al. 2009) confirmou as diferenças entre grupos encontradas por meio do IMC. Verificou-se uma relação entre o IMC dos participantes e a escolha da silhueta que representa Barbarói, Santa Cruz do Sul, n. 56, p.<164-188>,jan./jun. 2020 
seu corpo atual. Ou seja, a maior parte das pessoas sem excesso de peso identificou-se assim na escala de silhuetas, bem como quase todos os participantes com excesso de peso assim se identificaram, demonstrando que não houve distorção da imagem corporal. Salienta-se que corpo humano é objeto de representações que interferem nos processos de perceber e avaliar o próprio corpo, as quais não se dão apenas a partir de um objeto que porta características físicas, mas também como parte do self e portador de múltiplos afetos (MORIN \& DANY, 2010), que interferem na forma como as pessoas se relacionam com o próprio corpo e com o corpo dos outros (JODELET, 2017).

Assim, pode-se dizer que os dois grupos não só apresentaram a constituição corporal significativamente diferente, mas percebem seus corpos de modos significativamente distintos no que se refere à constituição corporal. As pessoas com excesso de peso, além de refletirem tal condição na silhueta percebida, também escolheram uma silhueta ideal menos magra que os demais. Uma imagem ideal mais magra que a considerada atual foi compartilhada pela maior parte dos participantes, o que revela certa norma corporal com referências que nem sempre condizem aos padrões de saúde (OMS, 2017).

Alguns autores referem os impactos diretos do material midiático e das redes sociais na autoimagem e satisfação corporal (LIRA, ET AL 2017; BESSENOFF, 2006). O presente estudo não procurou identificar tais efeitos. Entretanto, constatou-se coerência entre as representações e normas difundidas nos meios midiáticos (JUSTO \& CAMARGO, 2017) e a imagem corporal expressa pelos participantes desta pesquisa. A norma que preconiza o corpo magro e a necessidade de emagrecimento mostrou-se massivamente presente e considera-se que a apropriação desta poderá ser melhor explorada a partir da compreensão das RS ligadas à obesidade expressas pelos participantes, de modo a aprofundar a leitura do fenômeno em questão.

No que se refere à identificação de uma pessoa gorda, a fala dos participantes evidencia que a gordura é algo que se vê. É uma marca corporal que está facilmente visível (FERREIRA \& MAGALHÃES, 2005; SUDO \& LUZ, 2007) e que remete ao conceito proposto por Tajfel (1981) quando este autor fala do processo de pensamento por estereótipos, ou categorização social. A partir de sua teoria, tende-se a organizar as pessoas em categorias, no lugar de tratálas como seres únicos. Há uma tendência a acreditar que todos os membros de uma mesma categoria seriam mais semelhantes entre si do que eles realmente são e mais distintos da categoria oposta, do que eles realmente são. Tais estereótipos teriam uma influência direta, tanto nos julgamentos quanto nas ações relativas ao grupo categorizado (BILLIG, 1984). No 
presente estudo, considera-se que tal processo de estereotipia tem influência direta nas RS construídas e expressas acerca da obesidade.

A teoria das RS, segundo Moscovici (1993), propõe-se a explicar o processo de pensamento das pessoas sobre o mundo. Objetiva-se compreender como as pessoas elaboram seus pensamentos e transformam as suas ideias em uma realidade que é percebida como "normal" (JODELET, 2015). Mais do que um agregado de informações, as representações possibilitam atribuir significado aos fatos cotidianos, e nesse processo de significação mesclam informações, crenças, posicionamentos, normas e valores.

A análise de conteúdo dos excertos de entrevista relativos à obesidade revelou uma RS organizada em três dimensões: Origem, Caracterização e Consequências, sintetizada na figura 1, a fim de que se possa melhor compreender as relações entre as categorias. As três dimensões em torno das quais a RS se apresentou nas entrevistas, revelam uma teoria de senso comum.

ORIGEM

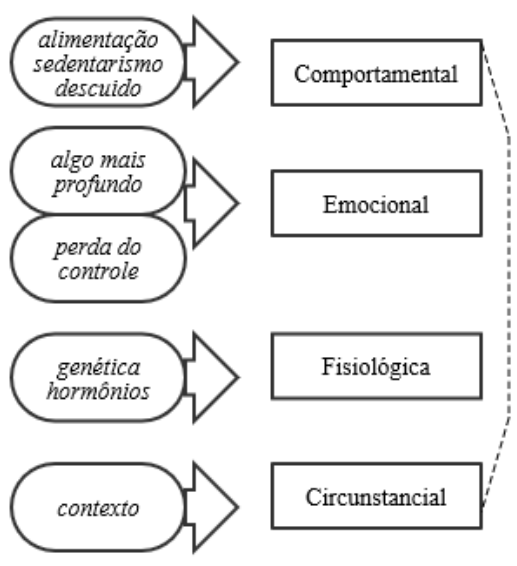

CARACTERIZAC̣̃̃o
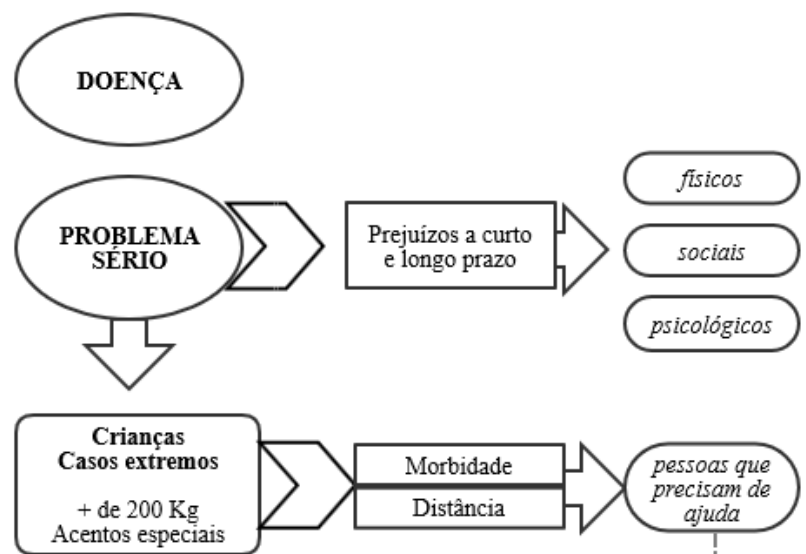

CONSEQUENCIAS

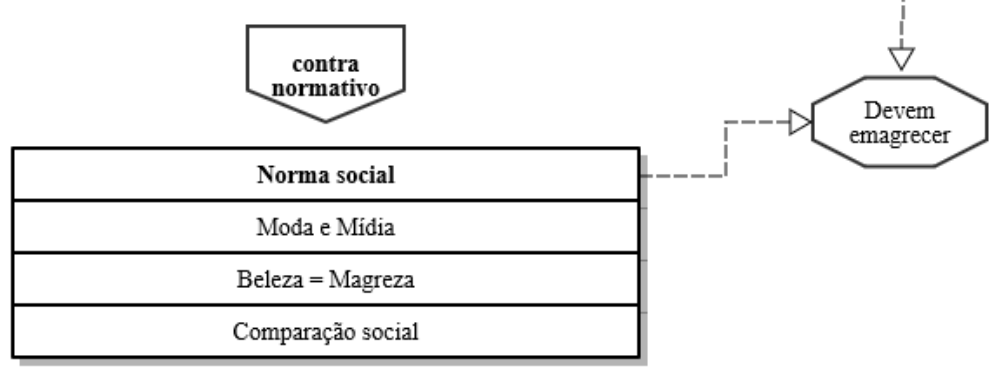

Figura 1. Modelo esquemático da RS da Obesidade, a partir da AC.

Fonte: Elaborada pelos autores

As representações envolvem atividades psíquicas por meio das quais realidade física e social se tornam inteligíveis (MOSCOVICI, 2012). E este processo atribuição de sentido, como aponta Moliner (2009), passa por saber "como" e "porquê" as coisas são como são, trazendo 
alguma sensação de previsibilidade à realidade vivida. Ou seja, a atribuição de causas pode ser considerado um processo sociocognitivo sucetível à elaboração de RS, de forma a ilustrar os processos de ancoragem e objetificação (BLAUDT; RANGEL, 2018; MOLINER, 2009).

O processo de ancoragem (JODELET, 1984) fica envidente, por exemplo, quando ao representar a obesidade, embora mencionem as causas fisiológicas e circunstanciais, os participantes enfatizam sua origem comportamental, que envolve má alimentação, sedentrarismo e até mesmo um "descuido" do próprio corpo. Moscovici (1993) afirma que a maior parte das pessoas atribuem causas a fatores internos e individuais, no lugar de fatores externos ou situacionais. Estudos clássicos sobre a percepção de grupos sociais alvo de preconceito demonstram que para estes tal tendência se acentua (SORGE, 2008), o que acaba refletindo numa responsabilização dos mesmos por sua condição. No mesmo sentido indica Joffe (2015) que a obesidade remete à falta de controle si, o que culmina em comummente e a pessoa obesa ser culpabilizada por sua condição.

Por sua vez, o processo de objetificação da RS (JODELET, 1984) mostra-se na imagem da obesidade evocada pelos participantes. Jodelet (2011) aponta que a distinção entre conhecimento e significado é essencial, sendo a maior contribuição de Moscovici, em que se destaca o caráter figurativo, imagético do pensamento de senso comum. Neste caso, a objetificação se concretiza em uma ideia caricata, associada às pessoas morbidamente obesas, que necessitam de espaços especializados, ou ainda à obesidade infantil.

Como consequência, nesse processo o indivíduo obeso é estereotipado, seja um adulto drasticamente acima do peso, ou a criança que sofre de discriminação na escola. Esta generalização simplifica, ao mesmo tempo em que dramatiza o objeto. Tal dramatização do discurso, como proposto por Goffman (2014), tem uma importância utilitária às relações sociais. Ao objetivar-se a obesidade em uma imagem exagerada, por vezes caricaturada, essa se torna um fenômeno distanciado da experiência cotidiana dos participantes. Ou seja, mesmo aqueles participantes com um IMC que caracteriza a obesidade e que se sentem acima do peso, não se consideram incluídos nessa categoria social.

Uma análise dos resultados a partir da abordagem dimensional permite que se identifique os três elementos propostos por Moscovici (2012). Além da figura caricata que ilustra o processo de objetificação, o campo representacional acerca da obesidade a associa a uma doença, ou um problema sério, de saúde física e mental. No que se refere à dimensão informação, os participantes ressaltam suas consequências negativas, principalmente no que se refere aos agravos à saúde, mobilidade e as dificuldades relações sociais, que podem ter implicações emocionais. Estas consequências foram mais salientes para os participantes com Barbarói, Santa Cruz do Sul, n. 56, p.<164-188>, jan./jun. 2020 
excesso de peso. Junto disso, destaca-se a necessidade de reverter a situação de obesidade, logo, emagrecer, com o suporte das normas que "defendem a saúde".

O pensamento natural classifica os acontecimentos e as coisas do mundo, separando-as entre bom e ruim. Quando se trata em doenças epidêmicas um padrão de pensamento semelhante fora identificado (JOFFE, 1998) ao que se constatou nesse estudo acerca da obesidade. Verifica-se uma resposta fortemente emotiva, em que os sujeitos associados à doença tendem a ser vinculados a várias formas de depravação. A saúde está associada à virtude e conduta correta, enquanto a doença está associada ao pecado. A autora refere-se tipicamente à Aids (JOFFE, 1998; 2015), porém podemos identificar um pensamento semelhante em relação à obesidade, que envolve comportamentos considerados fora do controle, excessivos e irracionais.

Nesse sentido, destaca-se a dimensão mais marcada nos resultados da pesquisa, dentro da abordagem dimensional: a atitude. Sugere Moscovici (2012) que o ponto de partida de toda a RS é a afetividade, que orientará as demais dimensões da representação (informação e campo), de modo que as pessoas só buscarão se informar em função um posicionamento tomado (MOSCOVICI, 2012). Nesse estudo, a atitude relativa à obesidade mostrou-se negativa. Tal constatação é hegemônica nas entrevistas. Além disso, parece consensual a menção relativa ao "gordo" na terceira pessoa. É quase que inexistente no discurso a identificação pessoal (gordo como uma condição minha). Ou seja: o gordo é ele. Esse distanciamento reflete a extrema pressão normativa, a tal ponto que as pessoas não podem identificar-se como "gordas" ou "obesas".

A obesidade é atribuída ao outro, ao "não-eu" e, portanto, deve permanecer como fenômeno estranho, ou afastado, pois veicula características opostas àquelas que exprimem o que é associado à identidade (JODELET, 2017). Em termos afetivos, tal deslocamento do "eu" para o "ele" implica um distanciamento em relação ao objeto em questão. Trata-se de um fenômeno que é sempre colocado a distância, afastado da realidade experiencial e do corpo vivido (JODELET, 2006) dos entrevistados.

Assim, ao tomar as estratégias de emagrecimento como um "tratamento de saúde", verifica-se que as pessoas tendem a não o adotar pois, ainda que sejam capazes de identificar sua silhueta com o IMC acima do peso, não se identificam como "gordos", já que estes são invariavelmente apontados como uma outra categoria social (eles). Conforme sugerem Deschamps e Moliner (2009), este fenômeno pode refletir o esforço para preservar ou promover sua autoestima, o que envolve comparações sociais ligadas invariavelmente aos casos de extrema obesidade.

Barbarói, Santa Cruz do Sul, n. 56, p.<164-188>,jan./jun. 2020 
Um resultado deste processo é o de que, embora a informação de que a obesidade é prejudicial à saúde seja compartilhada de maneira unânime entre os participantes, inclusive entre os obesos, os mesmos não se percebem em risco, uma vez que o risco está no outro (ele). Nesse sentido, podemos observar a relação entre as três dimensões de uma RS (Moscovici, 2012), já que a dimensão atitudinal, ou seja, a avaliação negativa faz com que o fenômeno seja projetado como externo ao indivíduo (JOFFE, 1998), contribuindo para a objetificação de um campo ou imagem da obesidade que a coloca em distância, fixando-se em estereótipos. Tal constatação vai ao encontro dos achados de Lima et al (2017) que, em revisão de literatura identificaram que a pressão social exercida pelos estereótipos negativos, alimentada e difundida pela mídia, pela sociedade, e até mesmo pelos familiares, acaba por contribuir ainda mais para o aumento de peso em pessoas obesas.

\section{Considerações finais}

Conforme Sá (1994) o estudo de RS deve envolver produto e processo ligados às práticas sociais, garantindo que o aspecto cognitivo da RS emerja efetivamente na vida social cotidiana. O corpo humano consiste em um objeto privado, materialização da individualidade e das experiências mais íntimas que se pode experimentar. Ao mesmo tempo, trata-se do instrumento de comunicação com o mundo e com as outras pessoas; portanto, por excelência, um objeto social. Um objeto cotidiano, que está na interface entre o individual e o social (JODELET, 2017) e que engloba um sistema de normas presentes no sistema social, envolvendo tanto questões de apresentação e imagem, quanto de saúde, mostra-se então um objeto propício para buscar relações entre representações e práticas sociais.

Ao abordar os participantes ao longo da coleta de dados constatou-se que o assunto proposto se tratava de um tema relevante para a maioria das pessoas. Todavia, não se pode deixar de mencionar a resistência inicial que alguns indivíduos apresentavam ao abordá-lo. Embora trate-se de um assunto a respeito do qual as pessoas estejam implicadas, seu caráter aversivo resulta na não identificação com essa condição. Reitera-se que dentro de uma perspectiva psicossocial, não é a partir do corpo real, ou físico; mas sim a partir do corpo simbólico que se estabelecem os pensamentos, os afetos e as ações.

Considera-se que o atual ideal de saúde, pautado prioritariamente na responsabilização individual, reitera a culpabilização do indivíduo acima do peso pela sua condição, que vem a fortalecer os estereótipos. Como consequência, ainda que sejam salientes os riscos de saúde atrelados à obesidade, a tendência é uma não identificação com essa condição, o que pode ter impactos diretos no aumento de peso na população. Nesse sentido, salienta-se a importância de 
recuperar o corpo em sua dimensão experiencial, que implica em necessidades, desejos e limites. Por isso, considera-se pertinente incluir parâmetros em relação aos modelos que são difundidos pelas mídias, assim como o combate aos estereótipos ligados aos sujeitos obesos. Uma flexibilização nos padrões (tanto estéticos quanto de saúde) permitirá uma experiência corporal de menor opressão, e com maiores condições de identificação e de ação frente ao ganho de peso.

\title{
OBESIDAD, REPRESENTACIONES Y CATEGORIZACIÓN SOCIAL
}

\section{Résumen}

El objetivo de esta investigación es describir las representaciones sociales (RS) y el proceso de categorización social relacionado con la obesidad, desde un enfoque cualitativo. Se realizaron entrevistas semi-directivas con 20 hombres y 20 mujeres, con y sin exceso de peso, y se aplicó una escala de siluetas para evaluar la autoimagen. El objetivo de esta investigación es describir las representaciones sociales (RS) y el proceso de categorización social relacionado con la obesidad, desde un enfoque cualitativo. Se realizaron entrevistas semi-directivas con 20 hombres y 20 mujeres, con y sin exceso de peso, y se aplicó una escala de siluetas para evaluar la autoimagen. El procesamiento de datos incluyó estadística descriptiva y relacional, con la ayuda de SPSS y análisis de contenido temático-categórico utilizando el software ATLAS TI, con la elaboración de categorías emergentes de los datos y su organización en ejes temáticos. Los resultados revelan que dependiendo de la constitución del cuerpo, hay diferentes SR y niveles de satisfacción con el cuerpo. La RS de la obesidad está organizada en torno de la origen, caracterización y consecuencias, que constituyen una teoría del sentido común sobre el fenómeno. La fuerte presión reguladora contribuye a la aparición de un RS excesivamente estereotipado. Como consecuencia, aunque los riesgos para la salud asociados con el exceso de peso corporal son prominentes, la tendencia es no identificarse con esta afección, que contribuye al aumento de peso en la población.

Palabras-clave: representación social, obesidad, categorización social.

\section{OBESITY, REPRESENTATIONS AND SOCIAL CATEGORIZATION}

\begin{abstract}
This study aims to identify and describe the social representations (SR) related to obesity, based on a qualitative approach. Twenty men and twenty women with and without excess weight participated and a semi-directive interview was conducted in order to know the participants' conceptions about obesity and the criteria related to their social categorization. A silhouettes scale was also used to evaluate the self-image. The data processing involved descriptive and relational statistics, with SPSS, and thematic-categorical content analysis by ATLAS TI, with categories elaborated from the data later organized in thematic axes. The results reveal that depending on the body constitution, there are different SR and levels of body satisfaction. The SR related to obesity is organized around origin, characterization and consequences, constituting a common sense theory about the phenomenon. The strong regulatory pressure contributes to the emergence of an excessively stereotyped RS. As a consequence, although the health risks associated with excess body weight are prominent, the tendency is not to identify with this condition, which contributes to weight gain in the population.
\end{abstract}

Key words: social representation, obesity, social categorization. 


\section{REFERÊNCIAS}

ALMEIDA, A. M. O., \& SANTOS, M. F. S. A teoria das representações socais. In C. V. TORRES \& E. R. NEIVA (Orgs). Psicologia Social: Principais temas e vertentes (p.287295). Porto Alegre: Artmed, 2011.

ANDRADE, S. S. Saúde e beleza do corpo feminino: algumas representações no Brasil do Século XX. Movimento, v. 9, n.1, p.119-143, 2003.

ANDRIEU, B. Corps. In B. ANDRIEU (Org.) Le dictionnaire du corps en sciences humaines e sociales (p. 103-104). Paris: CNRS Editions, 2006.

ARAÚJO L. S.; COUTINHO M. P. L.;ARAÚJO-MORAIS L. C.;SIMEÃO S. S. S.; MACIEL S. C. Preconceito frente à obesidade: representações sociais veiculadas pela mídia impressa. Arquivos Brasileiros de Psicologia,v.70, n.1, p. 69-85, 2018.

BACKSTROM, L. From the Freak Show to the Living Room: Cultural Representations of Dwarfism and Obesity. Sociological Forum, v.27 n. 3, p. 682-707, 2012.

BANFIELD, S. S.; MCCABE, M. P. An Evaluation of the Construct of Body Image. Adolescence, v.37, n. 146, p. 373-393, 2012.

BARDIN, L. Análise de Conteúdo. Lisboa: Edições 70, 2009.

BERGER, P. L., ;LUCKMANN, T. A construção social da realidade. Petrópolis: Vozes, 1974.

BESSENOFF, G. R. Can the media affect us? Social comparison, self-discrepancy, and the thin ideal. Psychology of Women Quarterly, v.30, n.3, p. 239-251, 2006.

BILLIG, M. Political ideology: social psychology aspects. In H. TAJFEL (Org.), The social dimension: European studies in social psychology (Vol. 2., p. 446-465). Londres: Cambridge University Press, 1984.

BLAUDT, V. L. ; RANGEL, M.. Diálogos exequíveis entre representações sociais e outros paradigmas da psicologia social. Psicologia \& Sociedade, v. 30, e184257, 2018. https://doi.org/10.1590/1807-0310/2018v30184257.

BRASIL. Ministério da Saúde. Rede organiza atendimento a doenças crônicas. Portal da Saúde, 2013. Disponível em:

http://portalsaude.saude.gov.br/portalsaude/impressao/9573/893/rede-organiza atendimento-adoencascronicas.html, acesso em 05/05/2013.

BRASIL. Vigitel Brasil 2018 - vigilância de fatores de risco e proteção para doenças crônicas por inquérito telefônico: estimativas sobre frequência e distribuição sociodemográfica de fatores de risco e proteção para doenças crônicas nas capitais dos 26 estados brasileiros e no Distrito Federal em 2018. Secretaria de Vigilância em Saúde, Departamento de Análise em Saúde e Vigilância de Doenças não Transmissíveis. Brasília: Ministério da Saúde, 2019. 
BROCHU, P. M.; ESSES, V. M. What's in a Name? The Effects of the Labels "Fat" Versus "Overweight" on Weight Bias. Journal of Applied Social Psychology, v.41, n. 8, p.19812008, 2011.

CAMARGO, B. V.; JUSTO, A. M ; JODELET, D. Normas, Representações Sociais e Práticas Corporais. Revista Interamericana de Psicología. v.44, n.3, p.456-464, 2010

CAMPOS, P. H. F. O dilema dos "herdeiros de Durkheim": classes, grupos ou instituições? In E. M. Q. O. CHAMON; P. A. GUARESCHI; P. H. F. CAMPOS. Textos e debates em representação social (p. 47-68). Porto Alegre: ABRAPSO, 2014.

CASTRO, I. R. R. Obesidade: urge fazer avançar políticas públicas para sua prevenção e controle. Cadernos de Saúde Pública, v.33, n.7, p.1-3, 2017.

DESCHAMPS, J.; MOLINER, P. A Identidade em Psicologia Social. Petrópolis: Vozes, 2009.

DOISE, W. Da Psicologia Social à Psicologia Societal. Psicologia: Teoria e Pesquisa, v.18 n.1, p.27-35, 2002.

FERREIRA, V. A; MAGALHÃES, R. Obesidade e pobreza: o aparente paradoxo. Um estudo com mulheres da Favela da Rocinha, Rio de Janeiro, Brasil. Cadernos de Saúde Pública, v.2, n.6, p.1792-1800, 2005.

GASKELL, G. Entrevistas individuais e grupais. In M. W. BAUER; G. GASKELL, (Orgs.) Pesquisa qualitativa com texto, imagem e som: um manual prático (p.64-89). Petrópolis: Vozes, 2002.

GHIGLIONE, R.; MATALON, B. O Inquérito: Teoria e Prática. Oeiras: Celta Editora, 1993. GOFFMAN, E. A representação do Eu na vida Cotidiana. Petrópolis: Vozes, 2014.

JESUÍNO, J. C. Estruturas e processos de grupo. Em: J. VALA; M. B. MONTEIRO (Orgs.) Psicologia Social (p. 293-331). Lisboa: Fundação Calouste Gulbekiann, 2002

JODELET, D. Répresentation Sociale : phénomènes, concept et théorie. In : S. MOSCOVICI (éd.), Psychologie sociale (p.363-384). Paris, P.U.F., 1984.

JODELET, D. Place de l'expérience vécue dans les processus de formation des représentations socials. In V. HAAS (Org.) Les savoirs du quotidien (p.235-255). Rennes: PUR, 2006.

JODELET, D. (2011). Returning to past features of Serge Moscovici's theory to feed the future. Papers on Social Representations, v.20, n,39, p.1-11, 2011

JODELET, D. Representações Sociais e mundos de vida Curitiba : PUCPRess, 2017.

JOFFE, H. Degradação, desejo e "o outro". In A. ARRUDA (Org.) Representando a alteridade (p. 109-128). Petropolis: Vozes, 1998. 
JOFFE, H. The self-control ethos. In G. SAMMUT; E. ANDREOULI, G; GASKELL,; J. VALSINER (Eds.), The Cambridge handbook of social representations (p. 369-384). Cambridge, UK: Cambridge University Press, 2015.

JUSTO, A. M.; CAMARGO, B. V. Sobrepeso e obesidade na mídia impressa: uma análise retrospectiva na revista Veja. Estud. pesqui. psicol., Rio de Janeiro, v. 17, n. 3, p. 817-839, dez. 2017.

KAKESHITA, I. S., SILVA, A. I. P., ZANATTA, D. P., \& ALMEIDA, S. S. (2009). Construção e Fidedignidade Teste-Reteste de Escalas de Silhuetas Brasileiras para Adultos e Crianças. Psicologia: Teoria e Pesquisa, v.25, n. 2, p.263-270, 2009

LEGENBAUER, T.; RÜHL, I.,; VOCKS, S. Influence of appearance related TV commercials on body image state. Behavior Modification, 32, p.352-371, 2008.

LIMA, C. T.; RAMOS-OLIVEIRA, D.; BARBOSA. Aspectos sociocognitivos da obesidade: estereótipos do excesso de peso. Psicologia, Saúde \& Doenças, v.18, n.3, p.681-698, 2017.

LIRA, A. G. ET AL. Uso de redes sociais, influência da mídia e insatisfação com a imagem corporal de adolescentes brasileiras. J. bras. psiquiatr., v.66, n.3, p.164-171, 2017.

LOPES, R. G. C. Imagem e autoimagem: da homogenidade da velhice para a heterogenidade das vivências. In: A. L. NERI (Org.), Idosos no Brasil: vivências, desafios e expectativa na terceira idade. São Paulo: Editora Fundação Perseu Abramo, Edições SESC, 2007.

MATTOS R. S.; LUZ, M. T. Sobrevivendo ao estigma da gordura: um estudo socioantropológico sobre obesidade. Physis Revista de Saúde Coletiva, v. 19, n.2, p.489507, 2009.

MOLINER, P. Attribution causale et représentations sociales. In P. RATEAU; P. MOLINER (Orgs.), Représentations sociales et processus sociocognitifs (pp. 69-84). Rennes, FR: Presses Universitaires de Rennes, 2009.

MORRIN M.; DANY, L. Image corporelle et estime de soi: étude auprès de lycéens francais. Buelltin de Psychologie, v.63, n.5, p.321-334, 2010.

MOSCOVICI, S. The Return of the Unconscious. Social Research, v.60, n.1, p.39-93, 1993.

MOSCOVICI, S. A psicanálise: sua imagem, seu público. Porto Alegre: Vozes, 2012. (Originalmente publicado em 1961).

MUHR, T. User's Manual for ATLAS.ti 5.0. ResearchTalk Inc: Long Island, 2004,

ORGANIZAÇÃO MUNDIAL DA SAÚDE - OMS. Obesity: preventing and managing the global epidemic. In OMS, Report of WHO Consultation on Obesity. Geneva: World Health Organization, 1997.

ORGANIZAÇÃO MUNDIAL DA SAÚDE - OMS. Overweight and Obesity in the Western Pacific Region. An Equity Perspective. Western Pacific: WHO, 2017. 
PEIXOTO, M. R. G.; BENÍCIO, M. H. D.; JARDIM, P. C. B. V. Validade do peso e da altura auto-referidos: o estudo de Goiânia. Revista de Saúde Pública, v.40, n.6. p.1075-1072, 2006.

PEIXOTO, M. R. G.; CORDEIRO, M. M.; FERREIRA, V. R.; CARDOSO, C. K. S.;

CRISPIM, P. A. A. Ganho de peso na vida adulta: preditor da hipertensão arterial? Cad.

Saúde Coletiva. v. 25, n.1 p.58-64, 2017. DOI: 10.1590/1414-462X201700010023

PUHL, R. M.; LATNER, J. D.; KLING, K. M.; LUEDICKE, J. Weight bias among professionals treating eating disorders: attitudes about treatment and perceived patient outcomes. International Journal of Eating Disorders, v.47, p.65-75, 2014.

SÁ, C. P. Sur les relations entre représentations sociales, pratiques socio-culturelles et comportement. Papers on Social Representations, v.3, p.40-46, 1994.

SCHILDER, P. A imagem do corpo: As energias construtivas da psique. São Paulo: Martins Fontes, 1999.

SORGE, J. Integration of the stereotype content model and implicit theories: a dynamic understanding of stereotyping against obese individuals. Honors Theses. Paper 682, 2008. Disponível em : http://scholarship.richmond.edu/honors-theses/682

STENZEL, L. M. Obesidade: O peso da exclusão. Porto Alegre: EDIPUCRS, 2002.

SUDO, N./ LUZ, M. T. O gordo em pauta: representações do ser gordo em revistas semanais. Ciência \& Saúde Coletiva, v.12, n.4, p.1033-1040, 2007.

TAJFEL, H. Human Groups and Social Categories: Studies in Social Psychology. Londres: Cambridge University Press, 1981.

TORRES, T. L.; CAMARGO, B. V.; BOUSFIELD, A. B. S. Estereótipos sociais do idoso para diferentes grupos etários. Psic.: Teor. e Pesq., Brasília, v. 32, n. 1, p. 209-218, Mar. 2016 .

WAGNER, W.; ELEJABABARRIETA, F.; LANSTHEINER, I. How the sperm domnates de ovum by metaphor in the social representation of conception? European Journal of Social Psychology, v.25, p671-688, 1995.

WOTT, C. B.; CARELS, R. A. Overt Weight Stigma, Psychological Distress and Weight Loss Treatment Outcomes. Journal of Health Psychology, v.15, n.4, p.60 -614, 2010.

Data de recebimento: 06/02/2020

Data de aceite: 09/06/2020 


\section{Sobre os autores:}

Ana Maria Justo é Professora Adjunta do Departamento de Psicologia da Universidade Federal de Santa Catarina. Endereço Eletrônico: justoanamaria@gmail.com

Brigido Vizeu Camargo é Professor Titular Emérito pelo Departamento de Psicologia e Professor do Programa de Pós-Graduação em Psicologia da Universidade Federal de Santa Catarina. Endereço Eletrônico: brigido.camargo@yahoo.com.br

Andréa Barbará Silva Bousfield é Professora Associada do Departamento de Psicologia e do Programa de Pós-Graduação em Psicologia da Universidade Federal de Santa Catarina. Endereço Eletrônico: andreabs@gmail.com 